Europhys. Lett., 43 (5), pp. 503-509 (1998)

\title{
Quantum rectifiers from harmonic mixing
}

\author{
I. GOYChUK and P. HÄNGGI \\ Institut für Physik, Universität Augsburg \\ Memminger Str. 6, D-86135 Augsburg, Germany \\ (received 6 April 1998; accepted in final form 13 July 1998) \\ PACS. 05.30-d - Quantum statistical mechanics. \\ PACS. $72.20 \mathrm{Ht}$ - High-field and nonlinear effects.
}

\begin{abstract}
We investigate dissipative quantum transport in extended periodic systems that are subjected to electric harmonic mixing fields $E_{\mathrm{hm}}(t)=E_{1} \cos (\Omega t)+E_{2} \cos (2 \Omega t+\varphi)$. Although such a drive possesses no net bias on average, the interplay of quantum dissipation and nonlinear response causes a finite directed current. We thus discover the paradigm of a dissipative quantum rectifier. The quantum current exhibits multiple reversals when driven in the nonadiabatic regime. As a function of temperature the quantum current displays a bell-shaped characteristic - constituting the benchmark for quantum stochastic resonance. Moreover, harmonic mixing also serves as a novel tool to selectively control quantum diffusion.
\end{abstract}

The constructive role of time-dependent external driving and inherent dissipation can produce a variety of unexpected phenomena such as fluctuation-induced directed current in periodic structures that lack reflection symmetry (ratchets) [1], or anomalous amplification of weak signals in threshold-like systems (stochastic resonance) [2]. Here, our focus will be on a quantum version of classical ratchet systems; an extension that has been studied only recently for the class of adiabatically rocked ratchets [3]. Such a generalization of classical ratchet work into the world of quantum mechanics is far from being straightforward - due to the necessity of treating dissipation and external driving within a quantum-mechanical setting. As has been demonstrated previously for classical systems, directed current is possible also in symmetric periodic potentials, when driven by unbiased forces with nonvanishing odd numbered cumulant averages of order $n \geq 3$ [4]. Such a particular realization appears by considering the harmonic mixing signal of two ac fields of angular frequencies $\Omega$ and $2 \Omega$ that drive overdamped noise-driven classical transport in a cosine potential [5]. The phenomenon has implicitly been experimentally observed by means of microwave harmonic mixing in one-dimensional organic conductors as early as in 1978 by Seeger and collaborators [6] (see fig. 1 therein). Current, or, equivalently, a finite voltage under open circuit condition $[5,6]$ (i.e. a nonzero stopping bias) emerges due to a nonlinear response to the unbiased harmonic mixing signal

$$
E_{\mathrm{hm}}(t)=E_{1} \cos (\Omega t)+E_{2} \cos (2 \Omega t+\varphi)
$$

with relative phase $\varphi$. However, these prior works $[5,6]$ did not discuss the rectification phenomenon, or the "ratchet effect" [1,4]; thus the concept of Brownian machinery has been 
overlooked. As we demonstrate with this work, this harmonic-mixing mechanism is also of profound importance for driving induced dissipative quantum transport in periodic multi-state systems, such as THz-driven superlattices [7].

Driven dissipative quantum transport has recently been studied theoretically for monochromatic periodic driving (i.e. $E_{2}=0$ in (1)) in refs. [8-10], and for symmetric random driving in [11], in the presence of an additional static dc-bias. In both cases the current-voltage characteristics exhibit an interesting behaviour on dissipation strength and - most strikinglythe phenomenon of a zero-bias negative differential conductance (sometimes termed "absolute negative conductance"). This latter effect has been experimentally observed in GaAs-AlGaAssuperlattice structures [7]. Here, we shall demonstrate that similar quantum systems can act as nonadiabatic quantum rectifiers when driven by harmonic mixing signals.

To start, let us consider tunneling of a charged particle (electron) among sites in a onedimensional lattice in the presence of an electric field $E_{\mathrm{hm}}(t)$. We restrict the analysis to a single-band tight-binding treatment. The Hamiltonian then reads

$$
H_{\mathrm{TB}}(t)=-\frac{\hbar \Delta}{2} \sum_{n=-\infty}^{\infty}(|n\rangle\langle n+1|+| n+1\rangle\langle n|)-e E_{\mathrm{hm}}(t) \hat{q}
$$

where $|n\rangle$ denotes the localized (Wannier) states, $\hbar \Delta$ is the tunneling coupling energy between neighboring states, and $\hat{q}=a \sum_{n} n|n\rangle\langle n|$ is the position operator for the particle on the lattice with period $a$. In the absence of driving, this Hamiltonian is the archetype for variety of physical quantum transport phenomena [12,14]. For instance, it could be used to describe the current in semiconductor superlattices [14], or in charge-transferring molecular chains [16].

The harmonic mixing signal in (1) constitutes the simplest kind of an asymmetric periodic field possessing a nonvanishing third moment $\overline{E_{\mathrm{hm}}^{3}(t)}=\frac{3}{4} E_{1}^{2} E_{2} \cos \varphi$. Here $\overline{(\ldots)}$ indicates the time average over the temporal period, $\mathcal{T}=2 \pi / \Omega$. The Hamiltonian in (2) has intensively been studied for the monochromatic case with $E_{2}=0$; it describes, e.g., the remarkable phenomenon of dynamical localization [13-15]. It cannot, however, yield net current for an initially localized particle - because there is no thermal bath that balances the generated heat power- even in the case of additional presence of a dc-field. Thus, to realize finite current one has to invoke a dissipation mechanism. We adopt here the conventional model of quantum dissipation, i.e. we couple the quantum particle bilinearly to a thermal bath of harmonic oscillators [12]:

$$
H_{\mathrm{B}}=\frac{1}{2} \sum_{i}\left[\frac{\hat{p}_{i}^{2}}{m_{i}}+m_{i} \omega_{i}^{2}\left(\hat{x}_{i}-\frac{c_{i}}{m_{i} \omega_{i}^{2}} \hat{q}\right)^{2}\right] .
$$

The environmental influence is fully captured by the Gaussian thermal force operator $\hat{F}(t)=$ $\sum_{i} c_{i} \hat{x}_{i}(t)$, possessing the complex-valued autocorrelation function

$$
\langle\hat{F}(t) \hat{F}(0)\rangle_{\mathrm{eq}}=\frac{\hbar}{\pi} \int_{0}^{\infty} J(\omega) \frac{\cosh \left(\hbar \omega / 2 k_{\mathrm{B}} T-i \omega t\right)}{\sinh \left(\hbar \omega / 2 k_{\mathrm{B}} T\right)} \mathrm{d} \omega .
$$

Here, $J(\omega)=\frac{\pi}{2} \sum_{i} \frac{c_{i}^{2}}{m_{i} \omega_{i}} \delta\left(\omega-\omega_{i}\right)$ denotes the bath spectral density. In the present work we focus on Ohmic friction with spectral density $J(\omega)=\left(2 \pi \hbar / a^{2}\right) \alpha \omega e^{-\omega / \omega_{c}}$, with cut-off frequency $\omega_{\mathrm{c}} \gg \Delta$ and dimensionless friction strength $\alpha$.

Let the quantum particle be localized initially at the origin $n=0$. To describe quantum transport, we study the mean particle position $\langle q(t)\rangle=a \sum_{n} n P_{n}(t)$ and the mean-squared position $\left\langle q^{2}(t)\right\rangle=a^{2} \sum_{n} n^{2} P_{n}(t)$, with $P_{n}(t)$ denoting the site populations. The relevant 
transport quantities of interest are the net quantum current

$$
j=e \lim _{t \rightarrow \infty} \frac{\langle q(t)\rangle}{t},
$$

and the quantum diffusion coefficient

$$
D=\frac{1}{2} \lim _{t \rightarrow \infty} \frac{\left\langle\delta q^{2}(t)\right\rangle}{t}
$$

where $\left\langle\delta q^{2}(t)\right\rangle=\left\langle q^{2}(t)\right\rangle-\langle q(t)\rangle^{2}$. We consider here only the incoherent (i.e. sequential) tunneling transport regime which predominantly describes the experimental situation [7]. As a consequence, the so-termed noninteracting blip approximation (NIBA) $[9,11,12]$ is valid, and can be invoked to derive the related generalized master equation (GME) that governs the non-Markovian time evolution of the site populations $P_{n}$. As discussed in [12], and confirmed numerically by real-time quantum Monte Carlo calculations in [17], the NIBA provides indeed very good results for Ohmic friction within the regime of incoherent quantum transfer. In the absence of driving, NIBA is valid for any temperature at moderate-to-strong dissipation $(\alpha>1)$, and for not too low temperatures at weak dissipation $(\alpha<1)[12,17]$. Moreover, in fast periodic fields NIBA should be justified even better than without driving [18]. The related driven GME reads $[9,11]$

$$
\begin{aligned}
\dot{P}_{n}(t)= & \int_{0}^{t}\left\{W^{(+)}(t, \tau) P_{n-1}(\tau)+W^{(-)}(t, \tau) P_{n+1}(\tau)-\right. \\
& \left.-\left[W^{(+)}(t, \tau)+W^{(-)}(t, \tau)\right] P_{n}(\tau)\right\} \mathrm{d} \tau,
\end{aligned}
$$

with forward $(+)$ and backward $(-)$ transition kernels given in NIBA by

$$
W^{( \pm)}(t, \tau)=\frac{1}{2} \Delta^{2} e^{-Q^{\prime}(t-\tau)} \cos \left[Q^{\prime \prime}(t-\tau) \mp \frac{e a}{\hbar} \int_{\tau}^{t} E_{\mathrm{hm}}\left(t^{\prime}\right) \mathrm{d} t^{\prime}\right]
$$

where

$$
Q^{\prime}(t)+i Q^{\prime \prime}(t)=\frac{a^{2}}{\hbar^{2}} \int_{0}^{t} \mathrm{~d} t_{1} \int_{0}^{t_{1}}\left\langle\hat{F}\left(t_{2}\right) \hat{F}(0)\right\rangle_{\mathrm{eq}} d t_{2}+i \lambda t
$$

Here $\hbar \lambda=a^{2} \int_{0}^{\infty} \mathrm{d} \omega J(\omega) / \pi \omega$ denotes the bath reorganization energy [19]. It is worth noting that precisely the same structure as in (7) holds true for the exact GME, as can be deduced from the analysis in [20]. Here, we have approximated the exact memory kernels to order $\Delta^{2}$, and yet we treat the field dynamics to all orders exactly.

From (7) we then obtain for the current

$$
\frac{\mathrm{d}}{\mathrm{d} t}\langle q(t)\rangle=a \int_{0}^{t} \Gamma^{-}(t, \tau) \mathrm{d} \tau
$$

and for the quantum diffusion

$$
\frac{\mathrm{d}}{\mathrm{d} t}\left\langle q^{2}(t)\right\rangle=a^{2} \int_{0}^{t} \mathrm{~d} \tau\left[\Gamma^{+}(t, \tau)+2 \Gamma^{-}(t, \tau)\langle q(\tau)\rangle / a\right],
$$

where $\Gamma^{ \pm}=W^{(+)} \pm W^{(-)}$. Due to the periodicity of the driving $E_{\mathrm{hm}}(t)$, the kernels $\Gamma^{ \pm}(t, \tau)$ can be expanded into a Fourier series, namely

$$
\Gamma^{ \pm}(t+\tau, t)=\sum_{n=-\infty}^{\infty} \gamma_{n}^{ \pm}(\tau) e^{-i n \Omega t}
$$


Upon solving (9) and (10) explicitly in terms of the Laplace transform $\tilde{x}(s)=\int_{0}^{\infty} e^{-s t}\langle q(t)\rangle \mathrm{d} t$ and $\tilde{y}(s)=\int_{0}^{\infty} e^{-s t}\left\langle q^{2}(t)\right\rangle \mathrm{d} t$, one finds

$$
\begin{aligned}
& \tilde{x}(s)=\sum_{m=-\infty}^{\infty} \frac{a \tilde{\gamma}_{m}^{-}(s)}{s(s+i m \Omega)}, \\
& \tilde{y}(s)=\sum_{m=-\infty}^{\infty} \frac{a^{2} \tilde{\gamma}_{m}^{+}(s)}{s(s+i m \Omega)}+\frac{2 a^{2}}{s} \sum_{m, l=-\infty}^{\infty} \frac{\tilde{\gamma}_{l-m}^{-}(s+i m \Omega) \tilde{\gamma}_{m}^{-}(s)}{(s+i m \Omega)(s+i l \Omega)},
\end{aligned}
$$

with $\tilde{\gamma}_{m}^{ \pm}(s)$ denoting the Laplace transform of $\gamma_{m}^{ \pm}(t)$. The long-time dynamics of $\langle q(t)\rangle$ and $\left\langle q^{2}(t)\right\rangle$ is determined from the Tauberian theorems. For the net current we readily obtain $j=e a \tilde{\gamma}_{0}^{-}(0)$. Furthermore, the long-time behaviour of the position variance $\left\langle\delta q^{2}(t)\right\rangle$ follows from (12), (13) as $\left\langle\delta q^{2}(t)\right\rangle \sim 2 D t+O(t)$, where $O(t)$ is a bounded oscillating term, and

$$
D=D_{\mathrm{M}}+\frac{2 a^{2}}{\Omega} \sum_{m=1}^{\infty} \frac{\operatorname{Im}\left(\tilde{\gamma}_{m}^{-}(0) \tilde{\gamma}_{-m}^{-}(i m \Omega)+\tilde{\gamma}_{0}^{-}(0)\left[\tilde{\gamma}_{m}^{-}(0)-\tilde{\gamma}_{m}^{-}(-i m \Omega)\right]\right)}{m}
$$

is the quantum diffusion coefficient. We denoted by $D_{\mathrm{M}}=\frac{1}{2} a^{2} \tilde{\gamma}_{0}^{+}(0)$ the result of the Markovian approximation in (10). Note that essential non-Markovian effects can appear due to the driving-induced coherence even if the tunneling dynamics is incoherent in the absence of driving. However, a detailed analysis shows that for the present case these effects are negligible, so that $D_{\mathrm{M}}$ serves as a good approximation. For the rectification current $j$ and the Markovian diffusion coefficient $D_{\mathrm{M}}$ we find in NIBA (cf. eq. (8)) the central results

$$
\begin{array}{r}
j=e a \Delta^{2} \int_{0}^{\infty} \mathrm{d} \tau \exp \left[-Q^{\prime}(\tau)\right] \sin \left[Q^{\prime \prime}(\tau)\right] \operatorname{Im}[\Phi(\tau)], \\
D_{\mathrm{M}}=\frac{1}{2} a^{2} \Delta^{2} \int_{0}^{\infty} \mathrm{d} \tau \exp \left[-Q^{\prime}(\tau)\right] \cos \left[Q^{\prime \prime}(\tau)\right] \operatorname{Re}[\Phi(\tau)],
\end{array}
$$

where

$$
\Phi(\tau)=\sum_{k=-\infty}^{\infty} J_{2 k}\left(2 \xi_{1} \sin (\Omega \tau / 2)\right) J_{k}\left(\xi_{2} \sin (\Omega \tau)\right) e^{-i k(\varphi+\pi / 2)}
$$

Here, $J_{n}(z)$ are the Bessel functions of the first kind, and $\xi_{1,2}=e a E_{1,2} / \hbar \Omega$ measure the field strengths. Although no static bias is present, the net current can be nonzero because $\operatorname{Im}[\Phi(\tau)]$ is different from zero if both $E_{1} \neq 0$ and $E_{2} \neq 0$. Thus, the current appears as a result of interplay of the driving $E_{\mathrm{hm}}(t)$ and dissipation. This rectification effect is robust and does not depend on the details of dissipation. This is clearly seen from the structure of (15) which expresses the quantum current $j$ via the equilibrium autocorrelation (4) of the dissipative force $\hat{F}(t)$ and the nonlinear response function $\Phi(\tau)$.

For the present case of Ohmic friction, the corresponding bath correlation $Q(t)$ in (15), (16) can be evaluated in analytical form; see, e.g., in $[9,11,12]$. The result for the numerically evaluated rectification current $j$ vs. field strength $E_{1}$ is depicted in figs. 1 (a) and 1 (b) for two different friction strengths $\alpha$. The current is maximal in the adiabatic limit $\Omega \ll \omega_{\mathrm{c}}$. Most importantly, we notice that the current exhibits multiple reversals at moderate-to-strong friction $\alpha$ when driven in the nonadiabatic regime. The temperature dependence of the rectification current is depicted in fig. 2. At small (adiabatic) driving frequencies $\Omega$ and strong dissipation the current exhibits a bell-shaped behaviour, which is the benchmark of quantum stochastic resonance (QSR) [2]. This QSR feature gradually disappears with 

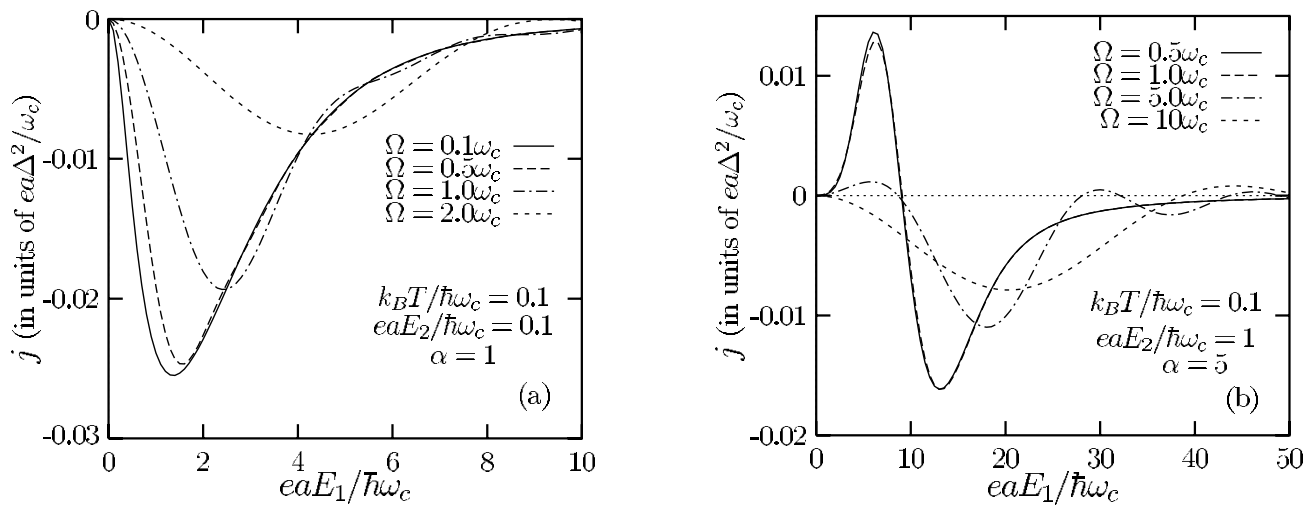

Fig. 1. - Net rectification current $j$ vs. scaled field amplitude $E_{1}$ for various field frequencies $\Omega$ and for two different Ohmic dissipation strengths: (a) $\alpha=1$ and (b) $\alpha=5$. The phase $\varphi=0$ is chosen.

increasing frequency $\Omega$ (nonadiabatic driving). It is absent also for weak dissipation. Notably is also the phenomenon of current reversal as a function of temperature when $k_{\mathrm{B}} T / \hbar \omega_{\mathrm{c}} \sim O(1)$. This phenomenon disappears however for $\Omega \gg \omega_{\mathrm{c}}$ (not shown).

We stress that the finite rectification current $j$ at zero external bias is a nontrivial nonlinear response phenomenon. In view of the fact that the dc-conductance is symmetric for a reflectionsymmetric, periodic quantum system, this result is remarkable indeed. To obtain more insight into the physical mechanism we assume that one of the harmonic mixing signals is weak, say $\xi_{2} \ll 1$. Then the series in (17) is well approximated by

$$
j=-e a \Delta^{2} \frac{e a E_{2}}{\hbar \Omega} \cos (\varphi) \int_{0}^{\infty} \mathrm{d} \tau \exp \left[-Q^{\prime}(\tau)\right] \sin \left[Q^{\prime \prime}(\tau)\right] \sin (\Omega \tau) J_{2}\left(2 \xi_{1} \sin (\Omega \tau / 2)\right) .
$$

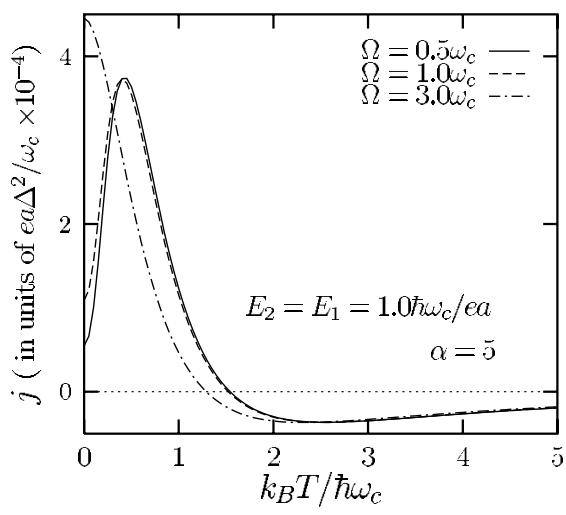

Fig. 2

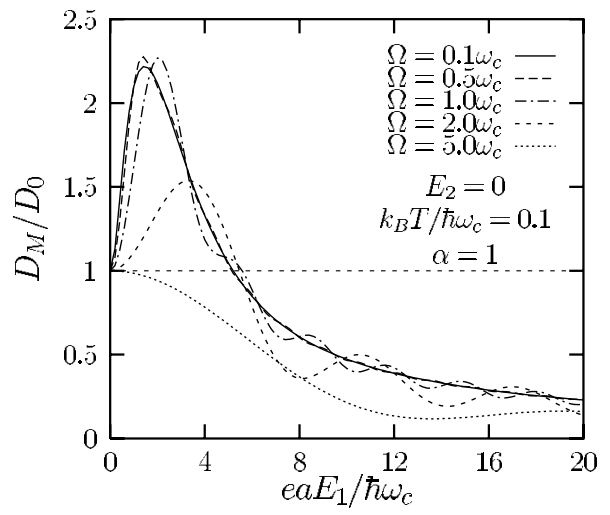

Fig. 3

Fig. 2. - Quantum stochastic resonance-like feature for harmonic mixing signals with $\varphi=0$ : The net rectification current $j$ exhibits a bell-shaped maximum vs. temperature $T$.

Fig. 3. - The scaled diffusion coefficient $D_{\mathrm{M}} v s$. field amplitude $E_{1}$ for various field frequencies $\Omega$. $D_{0}$ is the diffusion coefficient obtained by setting $E_{\mathrm{hm}}(t)=0$. 
As is seen from (18), the current in this approximation is linear in the strength of the second harmonic $E_{2}$. Hence the second harmonic creates an effective bias. Moreover, this result also shows that the phase $\varphi$ can be used to selectively control the strength of the rectification effect and the current direction. If in addition $\xi_{1}$ is also weak one finds from (18) that $j \propto \overline{E_{\mathrm{hm}}^{3}(t)}=\frac{3}{4} E_{1}^{2} E_{2} \cos \varphi$, with a nontrivial quantum prefactor. Thus, the phenomenon is rooted in the leading 3rd-order nonlinear response.

The selective control of quantum diffusion is depicted in fig. 3 for the case of zero rectification effect with $E_{2} \equiv 0$. The quantum diffusion coefficient $D_{\mathrm{M}}$ has been normalized to the nondriven quantum diffusion coefficient $D_{0}$, obtained by setting $E_{\mathrm{hm}}(t)=0$. As can be deduced from fig. 3 , the quantum diffusion can either be enhanced or suppressed by harmonic driving. The effect of enhancement is particularly interesting: With $\alpha \geq 1$, the dynamics is completely localized in the absence of driving at $T=0$, yielding $D_{0}=0$ [21]. The "switch-on" of an ac-drive will restore a finite quantum diffusion. The ac-drive thus destroys the dissipationinduced localization phenomenon $[12,21]$. The relative phase of the second-harmonic driving field in (1) allows one to selectively control the direction (and magnitude) of the rectification current $j$, and the strength of the quantum diffusion $D$ as well. In contrast to the case of net current $j$, cf. (18), the phase control of quantum diffusion occurs at second order of $E_{2}$.

Next we address the experimental realization. Our novel results should be observable in THz-driven superlattices [7,8]. Given a typical superlattice period $a \sim 200 \AA$ in GaAsAlGaAs superlattices, the miniband width $\Delta$ is estimated as $\hbar \Delta \sim 0.5-2 \mathrm{meV}[7,22]$ and the interband distance is of the order of $100 \mathrm{meV}$ [22]. The used $\mathrm{THz}$ frequencies are around $\hbar \Omega \sim 2-5 \mathrm{meV}$. With 20-50 photons needed to bridge the interband distance, our single-band approximation is a valid approximation scheme. A reasonable estimation for the cut-off frequency is $\hbar \omega_{\mathrm{c}} \sim 5 \mathrm{meV}$. The strongest field strengths, see, e.g., fig. 1(a), thus correspond to $E_{1} \sim 25 \mathrm{kV} / \mathrm{cm}$ and $E_{2} \sim 2.5 \mathrm{kV} / \mathrm{cm}$, being realistic values used in actual experiments for ac-driven photon-assisted tunneling (PAT) [7,8]. The maximal current in fig. 1(a) is around $200 \mathrm{nA} \cdot \AA$. Given an active area $S$ in a superlattice of $S=8(\mu \mathrm{m})^{2}$ and a carrier density $n=3 \times 10^{15} \mathrm{~cm}^{-3}$ [7], the predicted rectification current is of the order $i_{\max } \sim 500 \mathrm{nA}$. Although our modelling oversimplifies the microscopic mechanisms for dissipation and in addition neglects the many-particle Coulomb-interaction effects, the predictions should, nevertheless, hold up against actual experimental testing. This assertion has additional support from the fact that many of the previous monochromatically driven PAT results could be explained within an independent particle theory $[7,8,14,22]$.

In summary, we have studied the onset of nonzero current in periodic dissipative quantum systems when driven by two ac electric fields with angular frequencies $\Omega$ and $2 \Omega$ (harmonic mixing signal). This system thus exhibits rectification, i.e. the current maintains identical sign when subjected to a (small) dc-bias of opposite sign. As such, this quantum rectifier is minimal in the sense that its ingredients are elementary: A single-band periodic quantum system and two signals of commensurable frequencies $\Omega$ and $2 \Omega$. Nevertheless, it exhibits a rich behaviour for quantum transport: These being - apart from rectification - the existence of multiple reversals, cf. fig. 1(b), the QSR feature in combination with a reversal vs. temperature, cf. fig. 2, and the possibility to selectively manipulate (via control of the phase $\varphi$ in (1), and choice of driving field parameters) the strength of both quantum diffusion and rectification current, as well as current direction. By use of the harmonic mixing signal technique the novel surprising features of this nonlinear quantum response rectification scenario are expected to be observable in THZ-driven superlattices [7] and also in harmonic mixing driven current-biased Josephson junctions. 
The authors thank M. GRIFoni and P. Reimann for valuable discussions. Financial support was provided (IG) by the Alexander von Humboldt-Stiftung and (PH) by the Deutsche Forschungsgemeinschaft (HA1517/-14-2).

\section{REFERENCES}

[1] Astumian R. D., Science, 276 (1997) 917; Jülicher F., Adjari A. and Prost J., Rev. Mod. Phys., 69 (1997) 1269.

[2] Gammaitoni L., Hänggi P., Jung P. and Marchesoni F., Rev. Mod. Phys., 70 (1998) 223.

[3] Reimann P., Grifoni M. and Hänggi P., Phys. Rev. Lett., 79 (1997) 10.

[4] Van den Broeck C. and Hänggi P., Phys. Rev. A, 30 (1984) 2730; Luczka J., Bartussek R. and HäNGGi P., Europhys. Lett., 31 (1995) 431; HÄNGGI P. et al., Europhys. Lett., 35 (1996) 315; Zapata I., Luczka J., Sols F. and HängGi P., Phys. Rev. Lett., 80 (1998) 829.

[5] Wonneberger W. and Breymayer H.-J., Z. Phys. B, 56 (1984) 241; Breymayer H.-J., Risken H., Vollmer H. D. and Wonneberger W., Appl. Phys. B, 28 (1982) 335.

[6] Seeger K. and Maurer W., Solid State Commun., 27 (1978) 603.

[7] Keay B. J. et al., Phys. Rev. Lett., 75 (1995) 4098; 75 (1995) 4102.

[8] Ignatov A. A., Renk K. F. and Dodin E. P., Phys. Rev. Lett., 13 (1993) 1996; Ignatov A. A., Schomburg E., Grenzer J., Renk K. F. and Dodin E. P., Z. Phys. B, 98 (1995) 187.

[9] Hartmann L., Grifoni M. and Hänggi P., Europhys. Lett., 38(1997) 497.

[10] Platero G. and Aguado R., Appl. Phys. Lett., 70 (1997) 3546.

[11] Goychuk I. A., Petrov E. G. and May V., Phys. Lett. A, 238 (1998) 59.

[12] Weiss U., Quantum Dissipative Systems, Ser. Modern Cond. Matter Phys., Vol. 2 (World Scientific, Singapore) 1993.

[13] Dunlap D. H. and Kenkre V. M., Phys. Rev. B, 37 (1988) 6622.

[14] Holthaus M. and Hone D. W., Philos. Mag. B, 74 (1996) 105.

[15] Pronin K. A., Bandrauk A. D. and Ovchinnikov A. A., Phys. Rev. B, 50 (1994) 3473; Ovchinnikov A. A. and Pronin K. A., to be published in Chem. Phys.

[16] Mikkelsen K. V. and Ratner M. A., Chem. Rev., 87 (1987) 113.

[17] Mak C. H. and Egger R., Phys. Rev. E, 49 (1994) 1997.

[18] Grifoni M., Sassetti M., Hänggi P. and Weiss U., Phys. Rev. E, 52 (1995) 3596.

[19] Goychuk I. A., Petrov E. G. and May V., Phys. Rev. E, 52 (1995) 2392.

[20] Grifoni M., Sassetti M. and Weiss U., Phys. Rev. E, 53 (1996) R2033.

[21] Schmid A., Phys. Rev. Lett., 51 (1983) 1506; Guinea F., Hakim V. and Muramatsu A., Phys. Rev. Lett., 54 (1985) 263.

[22] Holthaus M., Z. Phys. B, 89251 (1992). 